\title{
Thrombosis and Bleeding as Presentation of COVID-19 Infection with Polycythemia Vera. A Case Report
}

\author{
Lai Chee Chow ${ }^{1}$ (D) $\cdot$ Lee Ping Chew ${ }^{1} \cdot$ Tze Shin Leong $^{1} \cdot$ Estrellita Elena Mohamad Tazuddin $^{2} \cdot$ Hock Hin Chua $^{3}$
}

Accepted: 21 September 2020 / Published online: 4 October 2020

(C) Springer Nature Switzerland AG 2020, corrected publication 2020

\begin{abstract}
Coronavirus disease (COVID-19) has a wide spectrum of clinical manifestations. In this case report, we describe our first case of COVID-19 pneumonia that was complicated by cerebral venous thrombosis and bleeding in a patient with polycythemia vera. Madam A, a 72-year-old lady with polycythemia vera, ischemic stroke, hemorrhoids, diabetes mellitus, hypertension, and dyslipidemia was admitted to the hospital for COVID-19 pneumonia. She was treated with hydroxychloroquine and lopinavir/ ritonavir as per hospital protocol. She continued taking hydroxyurea and aspirin for her treatment of polycythemia vera. Subsequently, she developed rectal bleeding when her platelet count was $1247 \times 10^{3} / \mu \mathrm{l}$, even though she was not on an anticoagulant. Her aspirin was withheld. One week later, she was readmitted to the hospital for cerebral venous thrombosis and her D-dimer was $2.02 \mu \mathrm{g} / \mathrm{ml}$. She was commenced on a therapeutic dose of low molecular weight heparin. Following that, her D-dimer level showed a decreasing trend and normalized upon her discharge. Patients with polycythemia vera are prone to develop thrombotic and bleeding complications. Management of this group of patients has become more complex with COVID19 infection. It is crucial for us to decide when to start an anticoagulant especially when there is a history of recent bleeding. We need to balance the risks of further bleeding versus potentially fatal thrombotic events. Studies have shown that D-dimer can be used as a clinical marker to predict thrombotic events in COVID-19 infection. Patients with COVID-19 infection and polycythemia vera will benefit from both pharmacological thromboprophylaxis and close monitoring for bleeding.
\end{abstract}

Keywords COVID-19 $\cdot$ Polycythemia vera $\cdot$ D-dimer $\cdot$ Cerebral venous thrombosis $\cdot$ Case report

\section{Introduction}

Coronavirus disease (COVID-19) was first detected at Wuhan, China, in December 2019. This ongoing global pandemic has caused millions of confirmed cases and thousands of deaths [1]. This disease has a wide spectrum of clinical manifestations, ranging from mild upper respiratory tract infection symptoms to severe pneumonia that require ventilation support, shock, and multi-organ failure [2]. COVID-19 is considered as multi-organ disease as it involves different organs and even systemic complications [3]. We report a case of an elderly patient with underlying polycythemia vera, diagnosed

This article is part of the Topical Collection on COVID-19

Lai Chee Chow

connie_chow2002@hotmail.com

Lee Ping Chew

leepingc@gmail.com

Tze Shin Leong

tzeshin2001@yahoo.com

Estrellita Elena Mohamad Tazuddin estee2020@yahoo.com

Hock Hin Chua

hhchua2009@gmail.com
1 Hematology Unit, Department of Medicine, Sarawak General Hospital, Ministry of Health Malaysia, Jalan Hospital, 93586 Kuching, Sarawak, Malaysia

2 Radiology Department, Sarawak General Hospital, Ministry of Health Malaysia, Jalan Hospital, 93586 Kuching, Sarawak, Malaysia

3 Infectious Disease Unit, Department of Medicine, Sarawak General Hospital, Ministry of Health Malaysia, Jalan Hospital, 93586 Kuching, Sarawak, Malaysia 
with COVID-19 pneumonia which was complicated by cerebral venous thrombosis. We obtained the written informed consent from her next of kin.

\section{Case Presentation}

Madam A, a 72-year-old lady, was diagnosed with JAK2 V617F mutation polycythemia vera in 2019 and was treated with tablet hydroxyurea $500 \mathrm{mg}$ daily and oral aspirin $75 \mathrm{mg}$ daily. She required therapeutic phlebotomy once a week for the first month as her hematocrit level was persistently more than $50 \%$. Prior to her diagnosis of polycythemia vera, she had a history of ischemic stroke in 2008 with residual left-sided hemiparesis, hemorrhoids with banding done twice, diabetes mellitus, hypertension, and dyslipidemia.

In early March 2020, during the COVID-19 outbreak in Malaysia, most of her family members whom she was staying with were infected with COVID-19. Therefore, she was screened for being a close contact and was tested positive for SARS-CoV-2 by qualitative real-time reverse-transcriptase-polymerase-chain-reaction (qRT-PCR) assay. She was admitted to the isolation ward for close observation as per local COVID-19 protocol. Her treatment of aspirin and hydroxyurea for her underlying polycythemia vera was continued. While under observation, she developed a worsening cough with chest radiograph showed worsening bilateral lower zone opacities. However, her oxygen saturation remained stable at 97 to $100 \%$ under room air. Due to the severity of her COVID-19 infection, she was commenced on oral hydroxychloroquine for 5 days and oral lopinavir/ritonavir for 2 weeks. Her clinical condition improved with treatment. However, she developed per rectal bleeding which was attributed to her hemorrhoids. Her hemoglobin was $16.6 \mathrm{~g} / \mathrm{dL}$, total white cell count was $25 \times 10^{3} / \mu \mathrm{l}$, and platelet count was $1247 \times 10^{3} / \mu \mathrm{l}$ at the time the bleeding occurred (Table 1 ). As a result, her hydroxyurea dosage was increased to
$500 \mathrm{mg}$ twice daily but her aspirin was withheld. Her rectal bleeding resolved after that. She was hospitalized for 40 days and was discharged following a negative COVID-19 swab. She was not commenced on any anticoagulant in view of the history of rectal bleeding.

One week post discharge, she was readmitted with sudden onset of altered consciousness associated with right-sided body weakness. Her Glasgow Coma Scale (GCS) was 14/15, E3V5M6 on presentation. Computed tomography (CT) brain was performed and revealed cerebral venous thrombosis of the straight sinus, vein of Galen, and bilateral internal cerebral veins with venous infarcts (Fig. 1). She was commenced on subcutaneous (S/C) enoxaparin $40 \mathrm{mg}$ twice a day (weight $42 \mathrm{~kg}$ ). On day 3 of admission, she had a repeat CT brain and cerebral venography for fluctuating GCS level. The repeat brain scan showed evidence of thrombosis involving the superior sagittal and right sigmoid sinus as well, bilateral vasogenic edema, swollen left basal ganglia, and left thalamic region. She was started on intravenous dexamethasone for 5 days to reduce cerebral edema and oral levetiracetam for seizure prophylaxis.

Her GCS started to improve 2 days after the commencement of the above treatment. She had a repeat non-contrasted CT brain a week after her 2nd CT brain and showed unchanged multifocal infarcts, no intracranial hemorrhage, and less swollen left basal ganglia region. At the time of her discharge, her GCS was 15/15 and she was on nasogastric tube feeding and wheel-chaired bound due to incomplete recovery of her motor function. On her clinic follow-up a month later, her D-dimer was normal at $0.24 \mu \mathrm{g} / \mathrm{ml}$, and her $\mathrm{S} / \mathrm{C}$ enoxaparin $40 \mathrm{mg}$ BD was converted to oral warfarin. She is clinically stable on her last review 3 months later.

\section{Discussion}

Patients with polycythemia vera are deemed high-risk group if aged more than 60 years old with or without
Table 1 Blood investigation results throughout hospital admission and follow-up

\begin{tabular}{lllllllll}
\hline & Day 9 & Day 42 & Day 43 & Day 53 & Day 54 & Day 67 & Day 82 & Day 96 \\
\hline $\mathrm{Hb}(\mathrm{g} / \mathrm{dL})$ & 15.3 & 16.6 & 15.2 & 14.8 & 14.5 & 15.5 & 14.4 & 14.8 \\
$\mathrm{HCT}(\%)$ & 49.2 & 53.3 & 46.9 & 47.1 & 46.4 & 46.3 & 43.8 & 45.2 \\
$\mathrm{TWCC}\left(\times 10^{3} / \mu \mathrm{l}\right)$ & 7.59 & 25 & 20.5 & 10.12 & 11.24 & 10.49 & 5.06 & 3.53 \\
Platelet $\left(\times 10^{3} / \mu \mathrm{l}\right)$ & 388 & 1247 & 978 & 489 & 497 & 505 & 415 & 284 \\
LDH $(\mathrm{U} / \mathrm{l})$ & 543 & 1021 & 843 & 516 & 540 & - & 356 & 369 \\
$\mathrm{PT}(\mathrm{s})$ & - & 13.8 & - & 12.3 & 12.3 & - & 12.2 & 12.2 \\
APTT $(\mathrm{s})$ & - & 48 & - & 43.3 & 43.9 & - & 50.4 & 35.8 \\
D-Dimer FEU $(\mathrm{ug} / \mathrm{ml})$ & - & - & 1.77 & 2.02 & 1.21 & 0.22 & 0.37 & 0.24 \\
Fibrinogen $(\mathrm{mg} / \mathrm{dL})$ & - & - & - & 352 & 365 & - & 548 & 409 \\
\hline
\end{tabular}




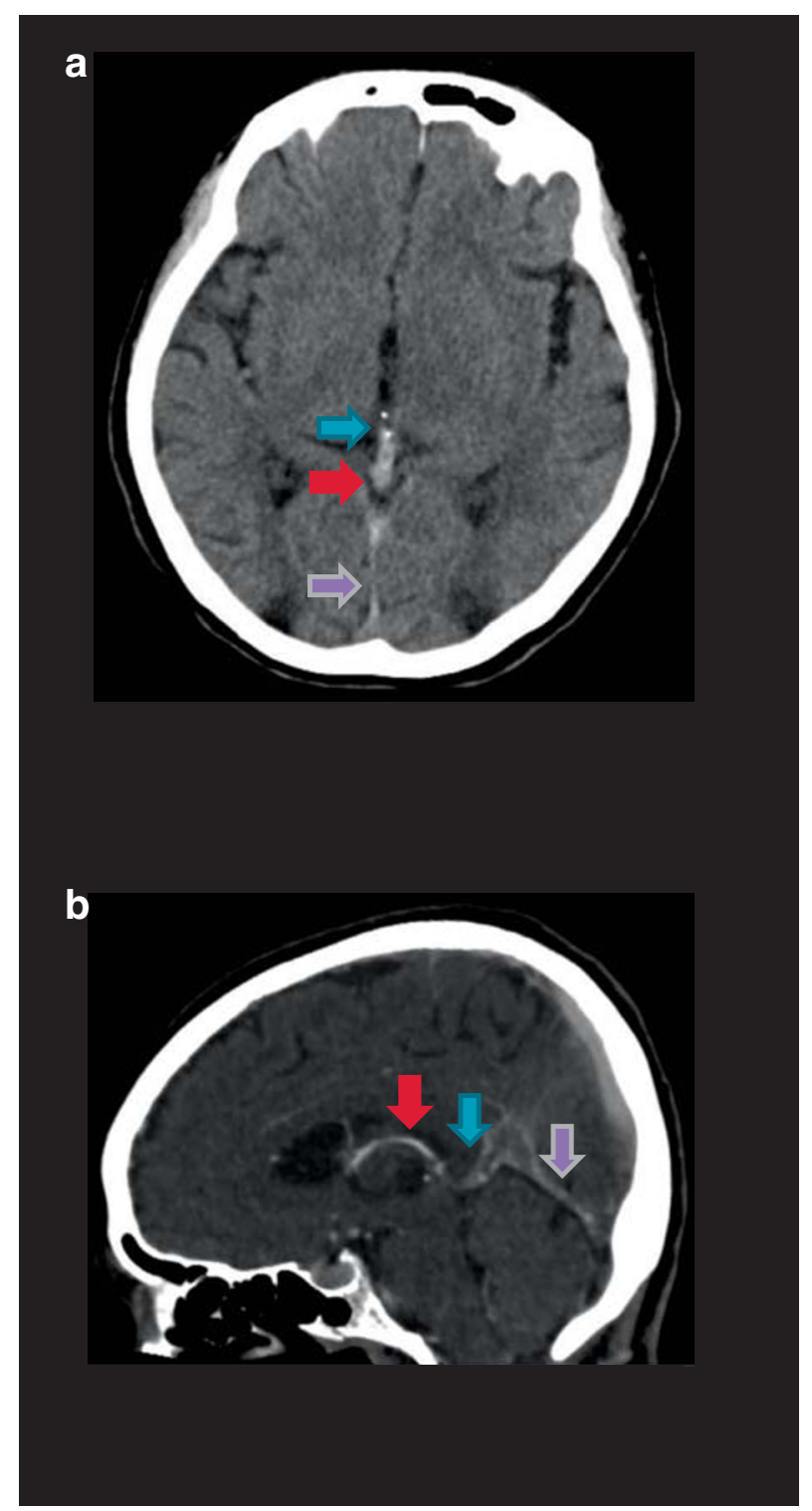

Fig. 1 a Axial non-contrast-enhanced CT showed ill-defined hypodensities at both basal ganglia and thalami, predominantly on the left, suggestive of the venous infarct. Hyperdense thrombus was seen in both internal cerebral veins $(\Rightarrow$ ) vein of Galen $(\vec{b})$ and straight sinus (
$\Rightarrow$ ). b Sagittal contrast-enhanced CT showed filling defect within the internal cerebral veins, vein of Galen, straight sinus, torcula herophili extending to the superior sagittal sinus history of thrombosis [4]. These patients with polycythemia vera are prone to develop thrombotic and sometimes bleeding complications. This is certainly the case for Madam A as she was 72 years old. Hence, she was treated with cytoreductive agent, hydroxyurea, and aspirin to achieve good disease control as per management of polycythemia vera in various guidelines [4-6].

However, management of this group of patients becomes more complicated with coronavirus infection. Coronavirus infection is a respiratory illness that was caused by SARSCOV-2. It releases pro-inflammatory cytokines such as IL-2, IL-6, and TNF-alpha which leads to systemic inflammatory response [7]. Patients with COVID-19 infection are postulated to be in a hypercoagulable state and prone to develop micro- and macrothromboses [7]. Several studies have shown an increase prevalence of thromboembolism in COVID-19 infection. In a series of 143 patients hospitalized with COVID-19 infection in China, $46.1 \%$ of them developed deep vein thrombosis [8]. In another study of 184 COVID19 ICU patients in the Netherlands, $31 \%$ of them had thrombotic complications despite receiving standard doses thromboprophylaxis [9].

We describe a case of cerebral venous thrombosis in COVID-19 infection with underlying polycythemia vera 


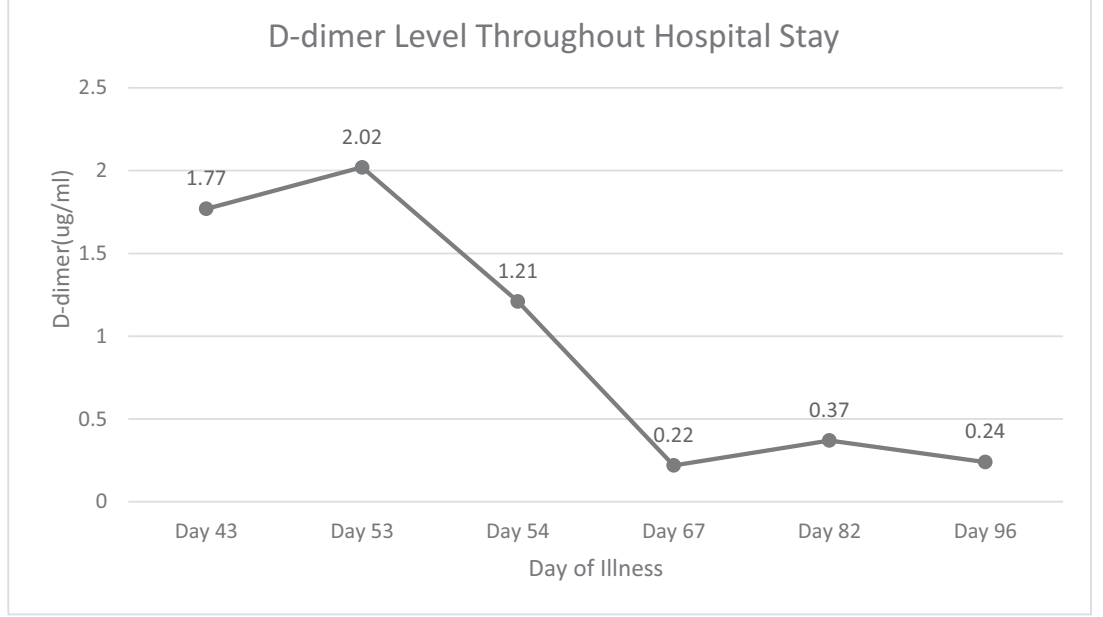

Graph 1 D-dimer level throughout the hospital stay and follow-up

that happened in Sarawak General Hospital, Malaysia during the early stage of the pandemic. To date, there is scarce data on patient with myeloproliferative neoplasm such as polycythemia vera, especially in the geriatric population, who has COVID-19 infection [10].

The complexity in the management of patient with polycythemia vera and coronavirus was demonstrated in the treatment of Madam A. Retrospectively, the patient should have been started on low molecular weight heparin while she was hospitalized based on prothrombotic tendencies of both coronavirus infection and polycythemia vera [11]. However, this was not implemented as there was no clear guideline during the early stage of a pandemic where the knowledge of coronavirus management was still limited. Furthermore, during Madam A's first admission, her platelet counts gradually increased to more than $1000 \times 10^{3} / \mu$ land she developed lower gastrointestinal bleed. We need to balance the risks of further bleeding versus potentially fatal thrombotic event.

To our knowledge, hypercoagulability in COVID-19 infection can manifest as an elevated D-dimer level. There are few studies that have shown patients with venous thromboembolism in COVID-19 infection have higher D-dimer [12, 13]. In this case, we did not have her baseline D-dimer level at the time of admission. Madam A's D-dimer level was $1.77 \mu \mathrm{g} / \mathrm{ml}$ $(<0.5 \mu \mathrm{g} / \mathrm{ml})$ when she had per rectal bleeding. Her D-dimer level was elevated from 1.77 to $2.02 \mu \mathrm{g} / \mathrm{ml}$ when she was diagnosed to have cerebral venous thrombosis during her second admission to the hospital (Graph 1). Her D-dimer level subsequently decreased after she was started on therapeutic dose of low molecular weight heparin and normalized upon her discharge and follow-up. This showed that D-dimer can be used to guide us in predicting thrombotic complication in COVID-19 infection.

However, there is a limitation of D-dimer usage as a clinical marker to predict thromboembolic events. Ddimer has high sensitivity but low specificity. It can be elevated in other medical conditions that increase fibrin production or breakdown. This includes malignancy, pregnancy, sepsis, myocardial infarction, stroke, liver disease, and postoperative state [14].

\section{Conclusion}

COVID-19 infection in a patient with polycythemia vera may present with bleeding and thrombotic manifestations. However, we should not treat this group of patients differently in the setting of coronavirus infection. Patients should be assessed accordingly. If they are deemed high risk of thrombosis when considering underlying diseases such as polycythemia vera, they should be started on pharmacological thromboprophylaxis with close monitoring of bleeding tendencies. This anecdotal experience could also lead us to consider using D-dimer as a surrogate clinical marker for the risk of developing thrombotic event. It can also be used to monitor response to treatment of thrombosis in polycythemia vera patients in the context of COVID-19 infection. However, this requires further validation via prospective studies.

Acknowledgments The authors would like to thank the Director General of Health Malaysia for the permission to publish this paper. We would like to thank our colleagues from COVID and infectious disease teams for their contributions and hard works during the COVID-19 pandemic.

\section{Compliance with Ethical Standards}

Conflict of Interest The authors declare that they have no conflict of interest.

Ethical Approval The study was carried out in accordance with the 1964 Declaration of Helsinki and subsequent amendments or comparable ethical standards.

Informed Consent Written informed consent was obtained from the patient's next of kin. 


\section{References}

1. World Health Organization (WHO) Coronavirus disease (COVID2019) situation report-209. https://www.who.int/docs/defaultsource/coronaviruse/situation-reports/20200816-covid-19-sitrep209.pdf?sfvrsn=5dde1 ca2. Accessed 17 August 2020.

2. Wu Z, McGoogan JM. Characteristics of and important lessons from the coronavirus disease 2019 COVID-19 outbreak in China: summary of a report of 72314 cases from the Chinese Center for Disease Control and Prevention. JAMA. 2020;323(13):1239-42. https://doi.org/10.1001/jama.2020.2648.

3. Spuntarelli V, Luciani M, Bentivegna E, Marini V, Falangone F, Conforti G, et al. COVID-19: is it just a lung disease? A case-based review. SN Compr Clin Med. 2020 Jul;28:1-6. https://doi.org/10. 1007/s42399-020-00418-6.

4. Griesshammer M, Kiladjian J, Besses C. Thromboembolic events in polycythemia vera. Ann Hematol. 2019;98:1071-82. https://doi. org/10.1007/s00277-019-03625-x.

5. Francesco P. How I treat polycythemia vera. Blood. 2012;120(2): 275-84. https://doi.org/10.1182/blood-2012-02-366054.

6. McMullin MF, Harrison CN, Ali S, Cargo C, Chen F, Ewing J, et al. A guideline for the diagnosis and management of polycythaemia vera. A British Society for Haematology Guideline. Br J Haematol. 184:176-91. https://doi.org/10.1111/bjh.15648.

7. Joly BS, Siguret V, Veyradier A. Understanding pathophysiology of hemostasis disorders in critically ill patients with COVID-19. Intensive Care Med. 2020;46:1603-6. https://doi.org/10.1007/ s00134-020-06088-1.

8. Zhang L, Feng X, Zhang D, Jiang C, Mei H, Wang J, et al. Deep vein thrombosis in hospitalized patients with COVID-19 in Wuhan, China: prevalence, risk factors, and outcome [published correction appears in circulation. 2020 Jul 14;142(2): e33]. Circulation. 2020;142(2):114-28. https://doi.org/10.1161/CIRCULATIONAHA.120.046702.

9. Klok FA, Kruip M, van der Meer N, Arbous MS, Gommers D, Kant KM, et al. Incidence of thrombotic complications in critically ill ICU patients with COVID-19. Thromb Res. 2020;191:145-7. https://doi.org/10.1016/j.thromres.2020.04.013.

10. Bentivegna E, Luciani M, Spuntarelli V, Speranza ML, Guerritore L, Sentimental A, et al. Extremely severe case of COVID-19 pneumonia recovered despite bad prognostic indicators: a didactic report. SN Compr Clin Med. 2020;29:1-4. https://doi.org/10.1007/ s42399-020-00383-0.

11. Pennica A, Conforti G, Falangone F, Martocchia A, Tafaro L, Sentimental A, et al. Clinical Management of adult coronavirus infection disease 2019 (COVID-19) positive in the setting of low and medium intensity of care: a short practical review. SN Compr Clin Med. 2020;29:1-6. https://doi.org/10.1007/s42399-02000333-w.

12. Demelo-Rodríguez P, Cervilla-Muñoz E, Ordieres-Ortega L, ParraVirto A, Toledano-Macías M, Toledo-Samaniego N, et al. Incidence of asymptomatic deep vein thrombosis in patients with COVID-19 pneumonia and elevated D-dimer levels. Thromb Res. 2020;192:23-6. https://doi.org/10.1016/j.thromres.2020.05.018.

13. Al-Samkari H, Karp Leaf RS, Dzik WH, Carlson J, Fogerty AE, Waheed A, et al. COVID-19 and coagulation: bleeding and thrombotic manifestations of SARS-CoV-2 infection. Blood. 2020;136(4):489-500. https://doi.org/10.1182/blood.2020006520.

14. Olson JD, Cunningham MT, Higgins RA, Eby CS, Brandt JT. Ddimer: simple test. Tough Problems Arch Pathol Lab Med. 2013;137(8):1030-8. https://doi.org/10.5858/arpa.2012-0296-CP.

Publisher's Note Springer Nature remains neutral with regard to jurisdictional claims in published maps and institutional affiliations. 